CrossMark \&lick for updates

Cite this: J. Anal. At. Spectrom., 2015, 30, 745

Received 31st October 2014 Accepted 16th December 2014

DOI: $10.1039 / c 4 j a 00384 \mathrm{e}$

www.rsc.org/jaas

\section{Neutron resonance transmission imaging for 3D elemental mapping at the ISIS spallation neutron source}

\begin{abstract}
Giulia Festa, ${ }^{\text {ab }}$ Enrico Perelli Cippo, ${ }^{c}$ Daniela Di Martino, ${ }^{* a}$ Roberta Cattaneo, ${ }^{a}$ Roberto Senesi, ${ }^{\mathrm{b}}$ Carla Andreani, ${ }^{\mathrm{b}}$ Erik Schooneveld, ${ }^{\mathrm{d}}$ Winfried Kockelmann, ${ }^{\mathrm{d}}$ Nigel Rhodes, ${ }^{d}$ Antonella Scherillo, ${ }^{d}$ Petra Kudejova, ${ }^{e}$ Katalin Biro, ${ }^{f}$ Krisztina Duzs, ${ }^{f}$ Zsuzsa Hajnal ${ }^{\mathrm{f}}$ and Giuseppe Gorini ${ }^{\mathrm{ag}}$

We demonstrate for the first time the viability of a three-dimensional (3D) elemental imaging technique based on Neutron Resonance Transmission Imaging (NRTI), which is a neutron technique based on the presence of a resonance structure in the neutron-induced reaction cross sections. These resonances allow the identification of elements and isotopes within an object in a non-destructive manner. A dedicated set-up on the INES (Italian Neutron Experimental Station) beamline of the ISIS spallation neutron source was employed for the experiments. An early mediaeval disc fibula from the Hungarian National Museum in Budapest was used for our demonstration. The methodology and analysis procedures are described and the results obtained from the reconstruction of the 3D NRTI elemental image of the ancient object are compared with the results obtained from other neutron-based 3D imaging techniques.
\end{abstract}

\section{Introduction}

Neutrons have been widely used in archaeometric research since 1960, soon after international projects on nuclear fission, with a growing number of innovative methods allowing non-destructive or non-invasive analysis. ${ }^{\mathbf{1 , 2}}$ Neutron techniques proved to be very suitable for the collection of information from the bulk of large cultural heritage objects. In fact neutrons penetrate through objects without substantial attenuation, which is a property that makes them ideal for non-destructive testing. In this context, the ANCIENT CHARM (Analysis by Neutron resonant Capture Imaging and other Emerging Neutron Techniques: new Cultural Heritage and Archaeological Research Methods) project, $^{3}$ funded by European Commission under the New and Emerging Science and Technology programme, developed non-invasive 3D

${ }^{a}$ Dipartimento di Fisica, Università degli Studi di Milano-Bicocca, Milano, Italy. E-mail: daniela.dimartino@unimib.it

${ }^{b}$ Dipartimento di Fisica, Università di Roma Tor Vergata, Roma, Italy. E-mail: giulia. festa@roma2.infr.it

'Istituto di Fisica del Plasma "Piero Caldirola"-CNR, Milano, Italy. E-mail: enrico. perelli@ifp.cnr.it

${ }^{d}$ STFC, Rutherford Appleton Laboratory, ISIS Facility, Chilton, UK

${ }^{e}$ Technische Universität Munchen, Heinz Maier-Leibnitz Zentrum (MLZ), Munich, Germany

${ }^{f}$ Hungarian National Museum, Budapest, Hungary

${ }^{g}$ CNISM, Unità di ricerca di Milano-Bicocca, Milano, Italy.E-mail: giuseppe.gorini@ unimib.it tomographic imaging techniques for elemental and phase composition detection for cultural heritage. Such techniques were based on established neutron measurements, including Neutron Resonant Capture Analysis (NRCA), Prompt Gamma Activation Analysis (PGAA) and Neutron Diffraction (ND). In this study, the new imaging technique Neutron Resonance Transmission Imaging (NRTI) is described, and a practical example of the analysis of a real archaeological sample is presented. NRTI has been previously applied successfully to the 2D elemental mapping of archaeological samples. ${ }^{4}$ This kind of analysis, which is based on neutron resonant capture, is particularly effective for samples composed of copper and its alloys because of the significant sensitivities for copper itself and for a range of elements such as $\mathrm{Ag}, \mathrm{As}, \mathrm{Sb}, \mathrm{Sn}$, and $\mathrm{Zn}$ (that are likely to be found in Cu alloys), and for Fe, which is also relevant for archaeological applications. A case study is described in this paper, pointing out the advantages as well as the limitations of this technique.

\section{Methods and data analysis}

\subsection{The principle of NRTI}

NRTI is a neutron technique based on the presence of a resonance structure in the neutron-induced reaction cross sections. ${ }^{5,6}$ Resonances are abrupt changes in the neutron absorption and/or scattering cross section of a nucleus, which result in the efficient removal of neutrons from the incident beam within a narrow energy band. The resonances of 
different isotopes are different; therefore, there is a particular fingerprint for a specific isotope, which allows the identification of elements and isotopes present in a sample. Neutron resonances occur over a wide energy range, but the most important for practical purposes are the ones in the so-called epithermal range (namely, between approximately $1 \mathrm{eV}$ and $1 \mathrm{keV}$ ). In NRTI, transmission spectra are obtained by measuring the Time Of Flight (TOF) of the epithermal neutrons transmitted through the object under investigation, as shown in Fig. 1. For a pulsed source, the energy $E$ of the neutrons is related to the TOF via the (non-relativistic) energy-time formula $E=1 / 2 m_{\mathrm{n}} L^{2} / \mathrm{TOF}^{2}$, where $m_{\mathrm{n}}$ is the neutron mass and $L$ is the distance between the source and the transmission detector. Resonances appear in the transmission spectrum as dips at characteristic energies as shown in Fig. 2. The dips used in our NRTI analysis are listed in Table 1.

\subsection{NRTI setup at INES beamline}

The NRTI measurements shown in this paper were performed at the INES (Italian Neutron Experimental Station) beamline of the ISIS spallation neutron source. ${ }^{7}$ INES is a neutron diffractometer viewing a water moderator at ambient temperature and provides a high epithermal neutron flux of about $10^{6} \mathrm{~s}^{-1} \mathrm{~cm}^{-2}$ at $100 \mathrm{eV} .^{8}$ The neutron detector used in the measurements (also developed in the framework of the ANCIENT CHARM project ${ }^{3}$ ) was a $10 \times 10$ array of $\mathrm{GS}_{20} 0^{6} \mathrm{Li}-$ enriched glass pixels, $2 \times 2 \times 9 \mathrm{~mm}^{3}$ each, arranged in a matrix with a pitch of $2.5 \mathrm{~mm}$, thus resulting in an active area of $25 \times 25 \mathrm{~mm}^{2}$. A detailed description of a prototype detector realized with the same principle, basically differing only in the number of pixels, is given elsewhere. ${ }^{9}$ In the case of the sample used as a case study (see paragraph 3.1), a set of 16 NRTI radiographs at different angles (i.e. 15 radiographs at $24^{\circ}$ intervals and a further $360^{\circ}$ radiograph) were collected. Because the total lateral dimensions of the sample were larger than the $25 \mathrm{~mm} \times 25 \mathrm{~mm}$ active area of the detector, four exposures were made for each angle (totalling in 64
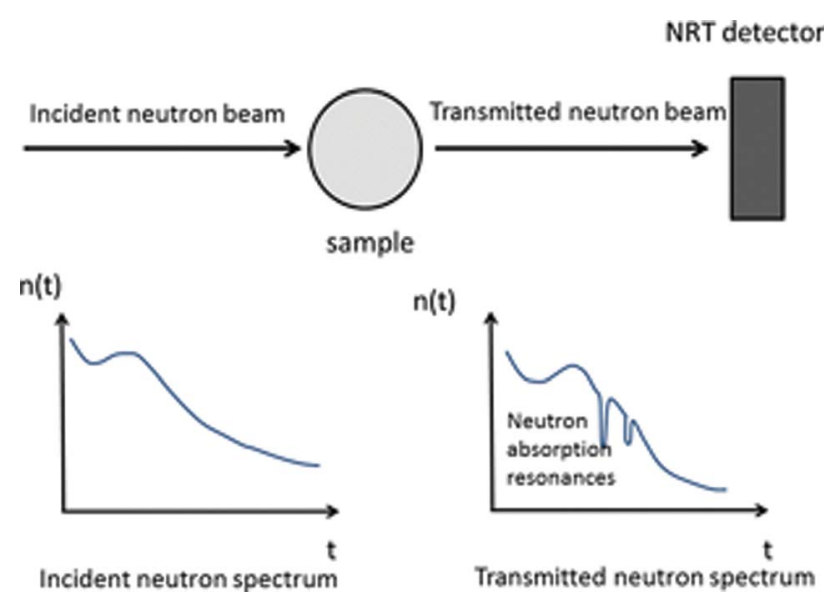

Fig. 1 Example of the set-up for a typical NRTI experiment.

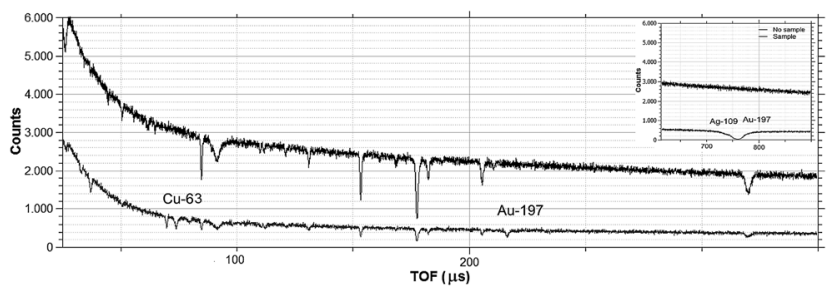

Fig. 2 Characteristic resonances dips in a neutron transmission spectrum.

exposures) and matched together, therefore resulting in each radiograph being composed of 256 NRTI spectra (in a $16 \times 16$ matrix).

\subsection{Data reduction and analysis in NRTI}

NRTI has been already used in $2 \mathrm{D}$ reconstruction imaging, after a feasibility study, based upon the comparison between a $2 \mathrm{D}$ image of a real object and that of a (purposely made) copy. ${ }^{4}$ In the present study, we have improved our previous research, extending the reconstruction process from a $2 \mathrm{D}$ visualization output to a $3 \mathrm{D}$ rendering of the same object. This extension is neither trivial nor certain. Moreover, our aim is to show that within acceptable time (with limited rotations and statistics), it is still possible to reconstruct a $3 \mathrm{D}$ elemental image from the sample.

As reported earlier, ${ }^{4}$ a rigorous route to analyse single NRTI spectra is based on multi-resonance shape analysis, which is a method implemented for instance in the REFIT code; ${ }^{\mathbf{1 0}}$ however, this procedure is very time consuming. In the case of NRTI tomography a large number of spectra need to be analysed to obtain 3D imaging information. As each pseudoradiograph is composed of 256 spectra, each pseudo-tomograph contains a very large number of resonance dips, which have to be fitted. Therefore, in this study, data were analysed following a different approach based on the assumption that the area of a transmission resonance dip is proportional to the concentration of the isotope that gives rise to the resonance dip. This hypothesis was discussed earlier ${ }^{4}$ and was assumed to be valid for small samples, of the order of a few centimetres, as is the case of the present sample. Single spectra were analysed in the OPEN GENIE software ${ }^{11}$ through ad hoc-developed routines. Such routines provided the following data reduction: (a) normalisation of the recorded spectra by the integrated ISIS proton beam current

Table 1 List of resonances used for the NRTI analysis

\begin{tabular}{lccr}
\hline Element & $E(\mathrm{eV})$ & TOF $(\mu \mathrm{s})$ & $\sigma(\mathrm{b})$ \\
\hline $\mathrm{Ag}$ & 5.2 & 736 & 11169 \\
$\mathrm{Cu}$ & 579 & 69 & 417 \\
$\mathrm{Au}$ & 4.9 & 760 & 27409 \\
$\mathrm{Fe}$ & 358 & 87 & 58
\end{tabular}


(following the standard normalisation procedure in use at ISIS $\dagger$ ); (b) fitting of the relevant dips using the least-squares method and an appropriate model. For the sake of simplicity, each dip was fitted with a simple Gaussian function in a TOF interval defined by the user. The background in such a limited interval was assumed to be linear, as displayed for the example in Fig. 3. The areas obtained using the fit procedures are reported in a two dimensional $16 \times 16$ matrix that could be associated with a pseudo-radiograph. These pseudoradiographs were built up for each detected element (in the present case $\mathrm{Ag}, \mathrm{Au}, \mathrm{Cu}, \mathrm{Fe}$ ) and represent the amount of each element crossed by the neutron beam in a specific direction. In order to obtain 3D maps of the elemental distribution inside the sample, a standard tomographic procedure was followed: a Filtered Back-Projection (FBP) algorithm, developed for X-ray tomography and implemented in the Imgrec software, ${ }^{12}$ was applied to each set of radiographs through a Matlab package. ${ }^{13}$ The results from the FBP were collected in the form of a $3 \mathrm{D}$ matrix, where each matrix element represents an attenuation pseudo-coefficient due to the presence of a specific resonance. Finally, the $3 \mathrm{D}$ matrices were transformed into 8 bit data, which is a format readable by commercial 3D visualization programs.

The main difference between such a 3D matrix and a standard neutron tomography matrix is that the attenuation pseudo-coefficient is valid for a specific energy range and that it can be positively ascribed to a specific atomic element. As a consequence, the resulting matrix is element-selective. ${ }^{12}$ In order to compare the resonances of different intensities on a similar scale, the different 3D matrixes were rescaled, taking as a reference the total area of the resonance cross section for each isotope. ${ }^{14}$ The number of rotations recorded during this experiment is lesser than the optimum number determined by the Nyquist criterion, usually a reference point for Neutron Tomography (NT), to guarantee the convergence of the FBP

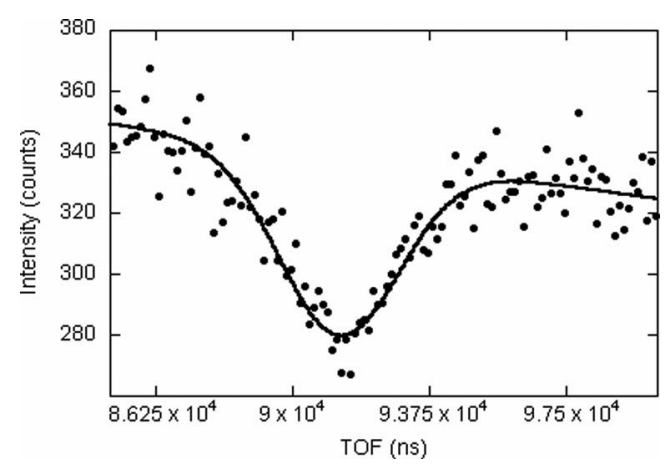

Fig. 3 Neutron resonance dip (experimental values as dotted symbols) fitted with a simple Gaussian function. The resulting fitted curve is displayed as a continuous line; the background in such a limited interval was assumed to be linear.

$\dagger$ In spallation neutron sources like ISIS the total proton charge on the spallation target during a measurement is proportional to the total number of neutrons impinging on the investigated sample. algorithm. ${ }^{12}$ This makes the experiment regarding the irradiation time requirements realistic.

\section{NRTI applied to an ancient artefact}

\subsection{The investigated sample}

The sample used to demonstrate the feasibility of NRTI is a mediaeval disc fibula (from the collection of the Hungarian National Museum in Budapest, Hungary) coming from Kölked-Feketekapu, one of the most important sites in the Carpathian basin, where an ancient Germanic population lived under the Avar rule. This type of fibula was commonly used along the River Rheine (by the Franks) and in Southern Germany (by the Alemanni). The fibula shows an iron band, which is quite unusual in this kind of object; moreover, it may be part of the original design or the result of a later restoration. ${ }^{15,16}$ A picture of this object, dated to the 6th century $\mathrm{AD}$, is displayed in Fig. 4.

\subsection{Results}

The results obtained by NRTI of the fibula can be summarized by the images shown in Fig. 5 and 6. Fig. 5, in particular, displays slices showing the concentration of copper, gold, silver and iron within the sample. These slices represent the amount

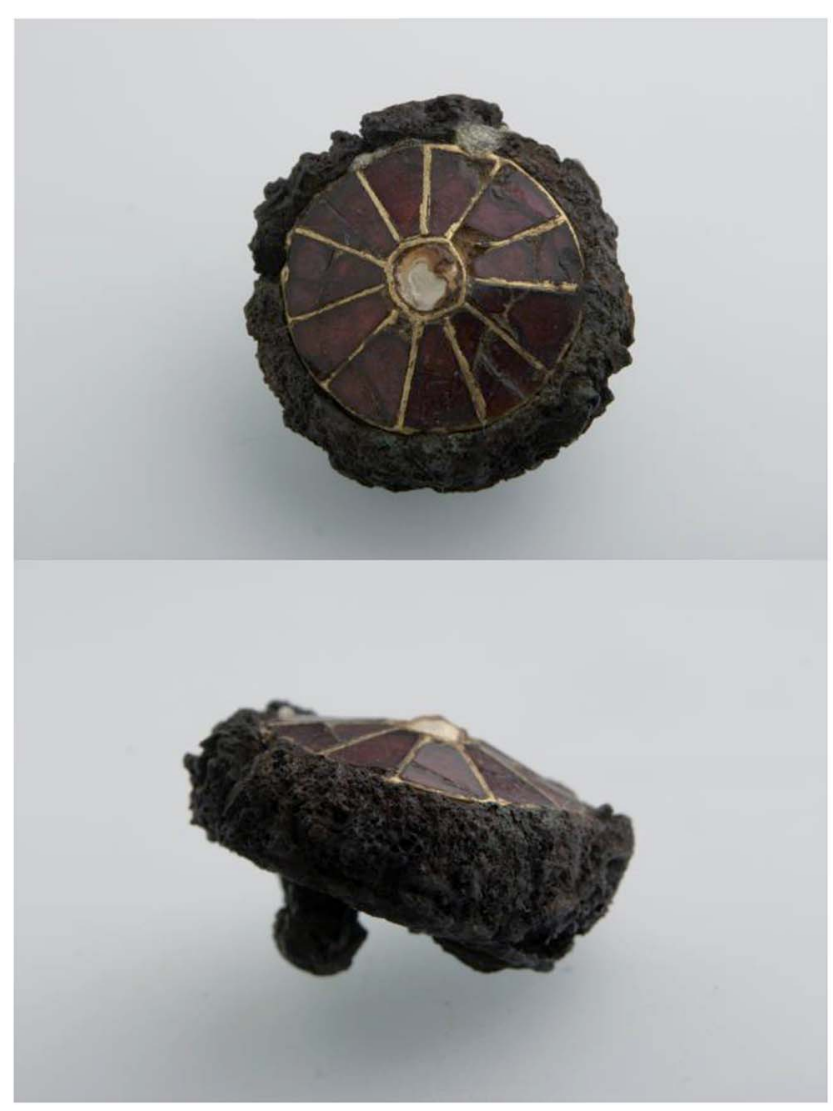

Fig. 4 Frontal and lateral pictures of the analysed disc fibula from Kölked-Feketekapu (Hungary), dated to the end of 6 th century AD. The diameter of the fibula is about $3 \mathrm{~cm}$. 


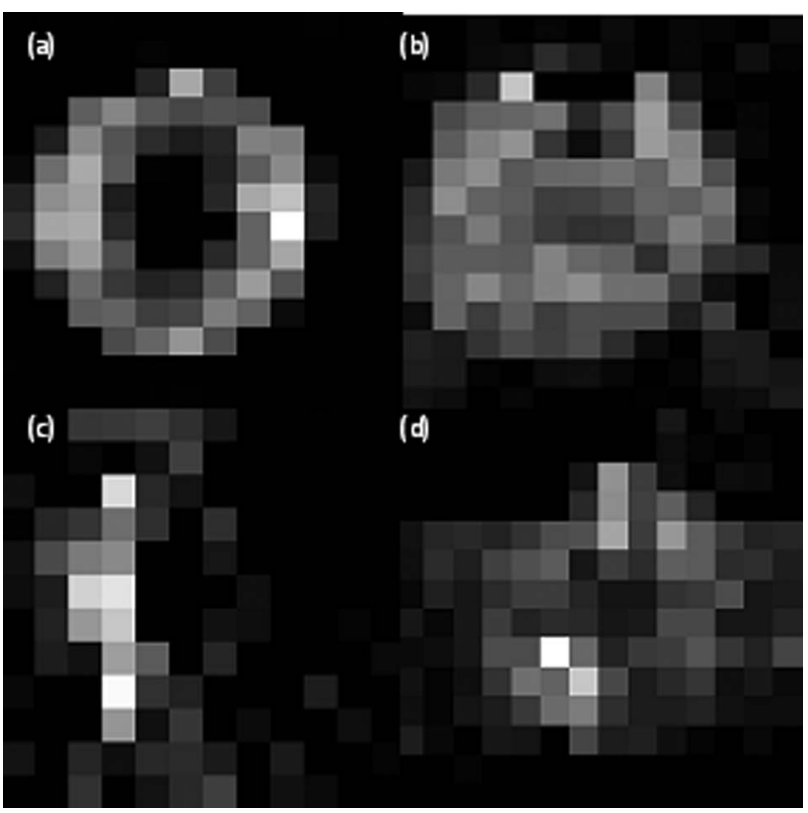

Fig. 5 Slice of copper (a), gold (b), silver (c), iron and (d) data sets obtained from the reconstruction process.
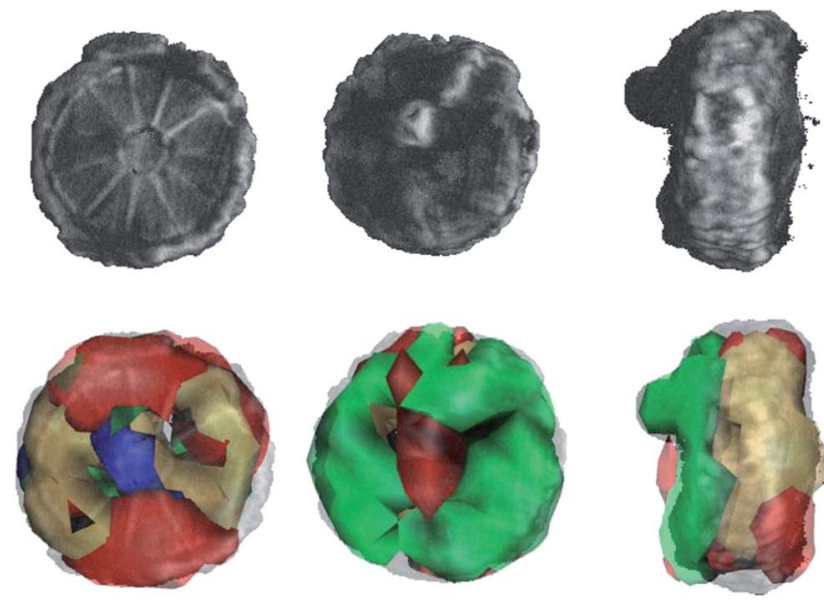

Fig. 6 Top part: Neutron tomography (previous result ${ }^{17}$ ). Bottom part $3 \mathrm{D}$ visualization of the elements in the fibula (isosurfacescolor map: $\mathrm{Cu}$ $=$ green, $\mathrm{Fe}=$ red, $\mathrm{Au}=$ yellow, $\mathrm{Ag}=$ blue) .

of a certain element present in the sample at a specific depth (the same for all slices). Fig. 6 shows the 3D isosurfaces of the spatial distribution of the elements superimposed over a neutron tomograph collected on the same object. Such a tomograph was recorded by Schulze et al. ${ }^{17}$ in the framework of the ANCIENT CHARM project ${ }^{3,18}$ along with elemental analysis collected through PGAI (Prompt Gamma Activation Imaging), i.e. 3D extrapolation of PGAA.

The results obtained by the two techniques are quite comparable. The distribution of the selected elements inside the object is as follows:

(a) Copper is basically present only on the rear of the sample, presumably originally constituting the back plate of the jewel. (b) Iron is mainly located on the ring structure around the main body of the fibula.

(c) Gold is present in the inlaying of the jewel; the silver distribution (although present in a very small quantity) quite likely follows the distribution of gold.

This kind of result, although only semi-quantitative, gives valuable indications to archaeologists because the inner parts of historical and artistic artefact could rarely be studied with other non-destructive techniques and they are sufficient, for instance, to argue about details of the construction technologies used. As far as point (c) is concerned, the presence of gold was not common for a fibula of that period. ${ }^{16}$ Moreover, the consistent occurrence of silver close to gold makes it more likely that the silver is an impurity in the gold alloy rather than the copper.

\section{NRTI compared to other 3D imaging techniques}

The graphical output of NT clearly shows a higher quality, in comparison with the NRTI 3D elemental image reconstruction (see Fig. 6) due to a limited spatial resolution of the latter $(2.5 \mathrm{~mm}$ compared to $50-100 \mu \mathrm{m}$ for NT). However, NT, though having a better spatial resolution, does not have a specific elemental sensitivity. It gives a clear representation of the hollow and solid parts of an object (for instance, for cultural heritage applications, a vase, case or other container, or a lost-wax statue), but it does not give an indication of the presence of a specific element, though a 3D mapping of the crystallographic phase distribution using energy-selective NT is possible. ${ }^{19}$ Recently, two studies were conducted on high resolution NRTI, ${ }^{20}$ obtaining a spatial resolution of $c a .150 \mu \mathrm{m}$, starting from 2D mapping. Although NRTI was not applied to a 3D real object, those studies confirm the interest in this non-destructive method.

As far as the two element-sensitive techniques are concerned, several differences and complementarities can be outlined between NRTI and PGAI. The number of elements that can be mapped with NRTI is lower than with PGAI, but this is not necessarily a limitation. For example, PGAI is sensitive to hydrogen, which is not visible in NRTI, but this element is not always relevant for archaeometric analyses. Currently, the two methods have the same spatial resolution of about $2.5 \mathrm{~mm}$. For NRTI, the spatial resolution can be improved by improving the resolution of the detector, ${ }^{20}$ whereas, for PGAI, the spatial resolution can only be improved by reducing the size of the neutron beam. This is much harder to accomplish and will considerably increase the measurement time; however, the most relevant practical difference is the time required for the measurements. PGAI measurements ${ }^{17}$ required 10 days of neutron irradiation for the analysis of a quadrant of the fibula; however, for NRTI, just 1 day was enough for recording the 64 radiographs needed for the reconstruction. This difference of more than an order of magnitude in time is very important considering two main points: (a) neutron measurements are very expensive and the required beam time should be reduced as much as possible, and (b) the irradiation time could be relevant for archaeometric objects at least in the case when 
lending time out of home collections/museums and insurance costs are a matter of concern.

Moreover, possible limitations of NRTI may arise from the nature and the dimensions of the samples to be investigated. With the present set-up (as described in Section 2.2) the total area of the NRTI-optimised detector is $25 \times 25 \mathrm{~mm}^{2}$, and at present, the practical application is limited to samples that have dimensions of the same order of magnitude. Larger samples may still be measured, taking into account that composite images and a higher number of rotations (thus requiring longer irradiation times) are needed. Another limitation is that not all elements show neutron resonances in the accessible energy range. As a result of both these points, a careful evaluation should be made by the investigators (and, in the case of cultural heritage objects, archaeologists and curators/restorers) in order to decide whether a specific sample is worth a NRTI analysis.

\section{Conclusions}

NRTI is a new non-destructive neutron-based technique for image reconstruction of the bulk elemental composition within a sample, which is a valuable result impossible to obtain with other conventional techniques. Bulk element concentrations can be determined using NRTI by placing an entire object in a neutron beam without any sample preparation. This makes NRTI especially useful in the cultural heritage field, where preservation of the investigated artefact is of paramount importance. For this reason, we have used an artefact as a case study to demonstrate the viability of this technique. NRTI was successfully applied to the 3D elemental reconstruction of an ancient disc fibula as explained in the previous sections.

Typical time for a complete NRTI tomography of objects of the dimensions of a fibula is of the order of 24 hours. Even with such a short irradiation time, the obtained results give important information to archaeologists and conservators. Elemental sensitivity is an important added value with respect to classical neutron tomography, while lower acquisition time is an important advantage compared to PGAI. ${ }^{17}$

\section{Author contribution}

G.F., E.P.C., G.G., R.S., R.C., C.A. conceived the idea of elemental imaging reconstruction through NRTI. P.K., K.B., K.D. and Z.H supplied the ancient artefact and all the archaeological information. E.P.C. performed the experiment assisted by A.S., E.S., W.K. and N.R.

G.F. and E.P.C. performed NRTI data reduction and analysis.

G.F., E.P.C. and D.D. analysed the results and wrote the manuscript in consultation with all the authors.

\section{Acknowledgements}

We acknowledge the financial support of the ANCIENT CHARM (Analysis by Neutron resonant Capture Imaging and other Emerging Neutron Techniques: new Cultural Heritage and Archaeological Research Methods) project, funded by European Commission under the New and Emerging Science and
Technology programme. This work was supported by the CNRSTFC Agreement no. 06/2008 concerning collaboration in scientific research at the ISIS spallation neutron source within the PANAREA project.

\section{Reference}

1 N. Kardjilov, et al., Neutron tomography for archaeological investigations, J. Neutron Res., 2006, 14, 29; W. Kockelmann, et al., Neutrons in cultural heritage research, J. Neutron Res., 2006, 14, 37.

2 E. H. Lehmann, P. Vontobel, E. Deschler-Erb and M. Soares, Non-invasive studies of objects from cultural heritage, $\mathrm{Nucl}$. Instrum. Methods Phys. Res., Sect. A, 2005, 542, 68.

3 G. Gorini, et al., Ancient Charm: A research project for neutron-based investigation of cultural-heritage objects, $I l$ NuovoCimento $C, 2007,47,30$, See also http://ancientcharm.neutron-eu.net/.

4 E. Perelli Cippo, et al., Imaging of cultural heritage objects using neutron resonances, J. Anal. At. Spectrom., 2011, 26, 992.

5 W. E. Lamb, Phys. Rev., 1939, 55, 190.

$6 \mathrm{H}$. Postma and P. Schillebeeckx, Encyclopedia of Analytical Chemistry, 2009, DOI: 10.1002/9780470027318.a9070.

7 S. Imberti, W. Kockelmann, M. Celli, et al., Meas. Sci. Technol., 2008, 19, 034003, See also: http://www.isis. stfc.ac.uk/instruments/ines/ines7450.html.

8 M. Celli, D. Colognesi, F. Grazzi, M. Zoppi, C. A. Checchi and E. M. Schooneveld, Not. CNR Neutroni e Luce di Sincrotrone, 2006, 11, 6 .

9 E. M. Schooneveld, et al., J. Phys. D: Appl. Phys., 2009, 42, 152003.

10 GEEL REFIT, A least squares fitting program for resonance analysis of neutron transmission and capture data computer code. M.C. Moxon, J.B. Brisland, AEA-InTec0630, 1991 (AEA Technology).

11 http://www.opengenie.org/Main_Page.

12 Developed by D. Schneberk at Lawrence Livermore National Laboratory. See also H. E. Martz, et al., X-ray Imaging, CRC Press Inc, Bosa Roca, 2009; A. C. Kak and M. Slaney, Principles of Computerized Tomographic Imaging, IEEE Press, Piscataway, NJ 08854 USA, 1988.

13 MATLAB, http://www.mathworks.it.

14 S. F. Mughabghab, Atlas of Neutron Resonances, Elsevier Science, 2006.

15 A. Kiss, Das awaren zeitlich gepidesche Graeberfeld von Koelked-Feketekapu, Studien zur Archaelogie der Awaren 5, Innsbruck, 1996, vol. 5.

16 G. Gorini and H. Kammermans, Conference: Proc. 14th International Congress Cultural Heritage and New Technologies (Vienna 2009), ed. W. Borner and S. Uhlirz, Museen der Stadt Wien - Stadtarchaologie, 2011.

17 R. Schulze, et al., The ANCIENT CHARM project at FRM II: three-dimensional elemental mapping by prompt gamma activation imaging and neutron tomography, J. Anal. At. Spectrom., 2013, 28, 1508. 
18 T. Belgya, Z. Kis, L. Szentmiklosi, et al., J. Radioanal. Nucl. Chem., 2008, 278, 751; P. Kudejova, et al., J. Radioanal. Nucl. Chem., 2008, 278, 691.

19 R. Woracek, et al., 3D Mapping of Crystallographic Phase Distribution using Energy-Selective Neutron Tomography, Adv. Mater., 2014, 26, 4069.
20 A. S. Tremsin, et al., IEEE Trans. Nucl. Sci., 2012, 59, 3272; A. S. Tremsin, et al., Nucl. Instrum. Methods Phys. Res., Sect. A, 2014, 746, 47 . 